\title{
THE EINSTEIN SOLID STATE SPECTROMETER AND MONITOR PROPORTIONAL COUNTER SURVEY OF LOW MASS X-RAY BINARIES
}

Damian J. Christian

Univ. of Maryland, Dept. of Astronomy, College Park, MD 20742 damian@astro.umd.edu

\begin{abstract}
The HEAO-2 Einstein solid state spectrometer (SSS; 0.5-4.5 keV) and monitor proportional counter (MPC; $1.2-20.0 \mathrm{keV}$ ) carried out an extensive survey of 50 low mass X-ray binaries (LMXB). Simultaneous SSS plus MPC spectra, selected on the basis of their intensity, were fit with a set of simple and complex spectral models. For all the sources, including Eddington-limited bulge sources, bursters, dippers, the soft spectrum black hole candidates, and a few transients in decline, the spectra could be fit acceptably with combinations of thermal bremsstrahlung and blackbody spectra or a Comptonized spectrum and a blackbody. The results rule out optically thick disk models for the bright (Z) sources and for the bursters power law models are unacceptable. The SSS can confirm only the strongest of previously reported low energy emission lines due to OVIII or Fe L transitions. The data does not support a unique physical interpretation.
\end{abstract}

\section{INTRODUCTION}

Low mass X-ray binaries (LMXB) are some of the most luminous galactic X-ray sources, and generally have high signal-to-noise spectra that have promised an explanation of their underlying physical processes. Only recently have high resolution data, more frequent and longer observations allowed a more complete understanding to come to fruition. Many LMXB exhibit dips, flaring with an increase in intensity of a factor of two, quasi-periodic oscillations (QPO), and X-ray bursts. LMXB can be simply classified as consisting of a class with luminosities of $\sim 10^{38} \mathrm{ergs} / \mathrm{s}$, and a less luminous class of a few 1036-5x1037 ergs/s (Parsignault and Grindlay 1978; Ponman 1982). EXOSAT observation have shown these sources are better classified on the basis of their color-color diagrams, the bright sources showing a strong pattern resembling a ' $Z$ ' with correlations to QPO and spectral behavior, and a group with less distinct patterns, the Atoll sources.

The large X-ray luminosities of LMXB are understood as accretion onto neutron stars from late type $\left(<1 \mathrm{M}_{\odot}\right)$ stars that have filled their Roche lobes. However, over the limited bandpass of many X-ray instruments, continuum models with very different physical interpretation have fitted the data equally well. The ambiguity in the spectral form of LMXB has arisen in part because counts at low energies are diminished by absorption by the interstellar medium, and high energy counts are lost due to lack of instrument effective area. The SSS and MPC of the Einstein observatory (HEAO-2) obtained simultaneous observations of 50 LMXB between November 1978 and October 1979 (January 1, 1979=JD 244 3873.5). This combination offers a bandpass of $0.5-20.0 \mathrm{keV}$ with the ability to test for soft components, measure the interstellar 
column density, and detect at least any strong low energy line emission or absorption features.

\section{Spectral Models}

\section{ANALYSIS}

Combined SSS and MPC spectra were fit with simple and complex continuum models. Fitting was performed with the 'Bspec' spectral fitting package (HEAO-2 analysis software), and Xspec. The photoelectric cross section for the absorbing column density along the line of sight was taken from work by Morrison and McCammon (1983). Spectral models included: a power-law (PL), blackbody (BB), optically thin thermal bremsstrahlung (TB), a physically thin and optically thick accretion disk model (Disk), an approximation to unsaturated Comptonization (USC; $\sim \mathrm{E}^{-}{ }^{-} \exp (-\mathrm{E} / \mathrm{kT})$ ), and Comptonization of the form of Sunyaev and Titarchuk (1980).

\section{RESULTS}

\section{Model Fits}

Single Component Fit to the SSS+MPC were generally poor for PL, BB, and Disk models. USC gave the best overall fits to the entire set.

Two component fits (addition of a BB) generally improved $\chi^{2}$ for all classes. The following points are notable. Bright sources are least well fit with $\mathrm{BB}+\mathrm{Disk}$ models. Bursters are least well fit with BB+PL. Almost all sources are reasonably well fit $\left(\chi^{2} \leq 2\right.$, corresponding to discrepancies of a few percent, which are not much more then remaining uncertainties in the response; Christian 1993) with BB+USC or BB+TB.

$\mathrm{BB}+$ Disk model parameters for fits to the bright $\mathrm{LMXB}$ often had disk temperatures of $0.7-1.5 \mathrm{keV}$ (soft component) and blackbody temperatures near $2 \mathrm{keV}$ (hard component). Mass accretion rates derived from the fits were not super-Eddington (0.2$5.5 \times 10^{17} \mathrm{gm} / \mathrm{s}$ ) as found by White, Stella, and Parmar (1988) for EXOSAT ME data. However, spectra show the disk model produces too few high energy counts to fit the data. This fact could only be determined for sources with good statistics (e.g Sco X-1 and GX 5-1), because of systematic errors of the MPC channels above $10 \mathrm{keV}$.

A two component model of $\mathrm{BB}+\mathrm{TB}$ has been found to be an adequate description of the data and provides a phenomenological model with which to compare the sources to other experiments (Swank and Serlemitsos 1985; Shulz, Hasinger, and Trumper 1989). Ranges of fit parameter are shown in Table 1. Many blackbody radii derived from the fits are near $10 \mathrm{~km}$ for the bright sources. The fractional contribution of the BB is plotted as a function of luminosity in figure 1. It shows the bright sources dominating at higher luminosities and BB fractions. Bursters typically have a contribution of less then $20 \%$.

Table 1. Typical BB+TB Fit Parameters

\begin{tabular}{|l|l|l|l|l|l|}
\hline Class & BB radius (km) & kT_BB (keV) & EM_60 cm-3 & kT_TB (keV) & \%BB \\
\hline Bright & $2-20$ & $0.8-2.6$ & $0.6-12.0$ & $5-10 \mathrm{keV}$ & $16-60$ \\
\hline Burster & $\sim 2$ & $2.0-2.5$ & $3.0-6.0$ & $7-10$ & $15-20$ \\
\hline Dippers & $1-14$ & $0.2-2.6$ & $0.1-1.0$ & $\sim 15$ & $10-99$ \\
\hline Soft & $3-30$ & $<1.3$ & $0.05-21.0$ & $<3$ & $30-70$ \\
\hline
\end{tabular}




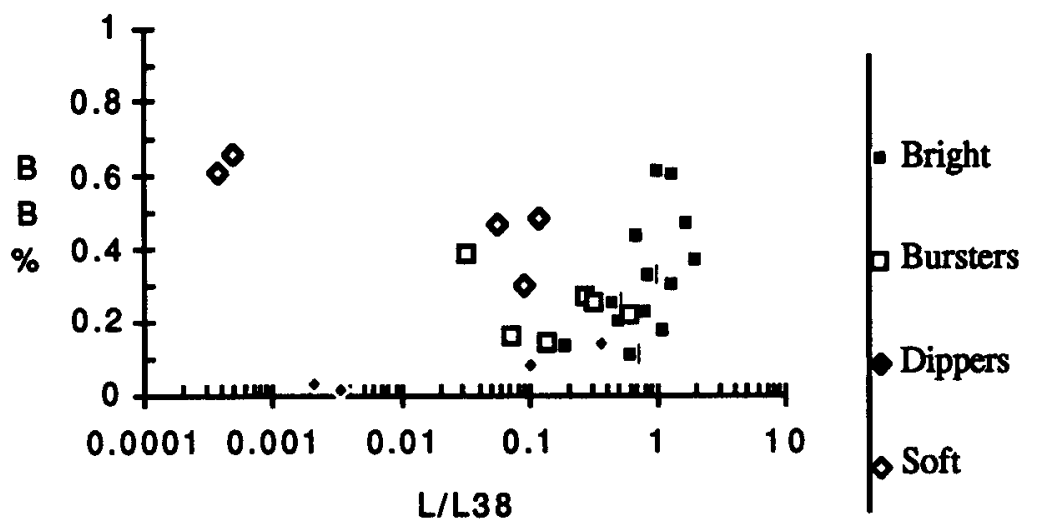

Figure 1. The percentage blackbody for the $\mathrm{BB}+\mathrm{TB}$ model plotted as a function of luminosity in unit of $10^{38} \mathrm{ergs} / \mathrm{s}$.

The USC model with $\Gamma=1.2$ to 1.4 is similar to a TB spectrum (i.e. TB with a Gaunt factor $\sim \mathrm{E}^{-0.4}$ in the $2-10 \mathrm{keV}$ range) and with $\Gamma=-2$ is a Wien spectrum. Thus with variations of $\Gamma$, USC can describe both an optically thin plasma and optically thick emission. The bright sources had $\Gamma$ ranging from -0.2 to 1.0 with cutoff temperature of 3-7 keV and luminosities ranging from $3 \times 10^{37} \mathrm{erg} / \mathrm{s}\left(\mathrm{GX} \mathrm{3+1)}\right.$ to $1.3 \times 10^{39} \mathrm{ergs} / \mathrm{s}$ (GX 5-1). The bursters have $\Gamma$ ranging from 1 to 2 with cutoff temperature of 5-10 $\mathrm{keV}$, and a wide range of luminosities (1036 ergs/s (0512-40) to $7.7 \times 10^{37} \mathrm{ergs} / \mathrm{s}$ (Ser $X-1)$ ). There are 4 soft sources in this sample with a variety of $\Gamma$ and cutoff energies less than $3 \mathrm{keV}$, and luminosities between $\sim 4 \times 10^{35} \mathrm{ergs} / \mathrm{s}(\mathrm{X} 0142+69)$ and $2 \times 10^{37}$ ergs/s (GX 339-4). Luminosity of the dippers range from $10^{34} \mathrm{ergs} / \mathrm{s}(4 \mathrm{U} 2129+47)$ to $10^{38} \mathrm{ergs} / \mathrm{s}(1624-49)$. $\Gamma$ is plotted as a function of luminosity in Figure 2.

\section{USC Model}
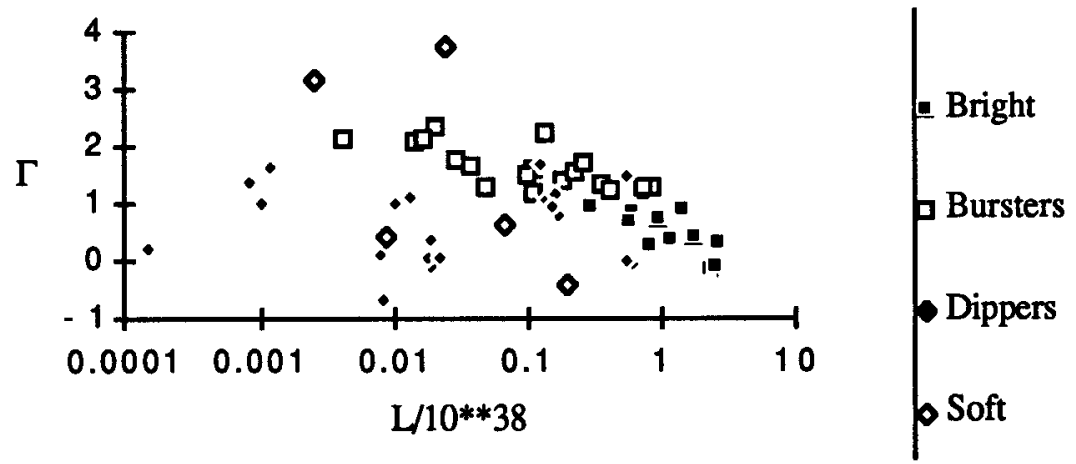

Figure 2. $\Gamma$ plotted as a function of luminosity for the USC model.

Fitting the data with the formal solution for the scattering of cool photons off hot electrons (Sunyaev and Titarchuk 1980) offered no improvement over the USC form with similar or slightly worse reduced $\chi^{2}$. Temperatures ranged from $1.1 \mathrm{keV}$ (X1755338 ) to $3.8 \mathrm{keV}$ (Cyg X-2), and moderately large optical depths (8 - 24). 


\section{Comparison with the EXOSAT ME}

BB+TB fits to time selected spectra of the bright LMXB were folded through the EXOSAT ME response matrix. This produced the counts that the ME would have observed in energy bands used by Shulz, Hasinger, and Trumper (1989) and the same softness and hardness ratios were generated. The states of the sources during the SSS+MPC observations then could be compared to the states observed with EXOSAT. Thus we determined GX 5-1 and GX 340+0 were on the horizontal branch, Cyg X-2 was on the normal branch, and Sco X-1, Sco X-2, GX 17+2, and an earlier Cyg X-2 observation on the flaring branch. Atoll sources, GX 9+9, GX 9+1, GX 3+1, and GX $13+1$ were found with generally larger softness ratios.

\section{Changes in Spectral Parameters}

For the bright sources we attempted to resolves temporal changes in terms of spectral model components or parameters. For example, the $\mathrm{BB}+\mathrm{TB}$ model changes in spectral parameters of Sco X-2 as it moved up the FB were complex. The fraction of blackbody contribution increased from 0.20 to 0.46 with changes in both $\mathrm{kT} \mathrm{BB}_{\mathrm{BB}}$ and $B B$ radius. The TB temperature and the TB emission measure were observed to be correlated with bremsstrahlung flux. GX $17+2$ showed similar FB behavior.

Low Energy Line Emission

The SSS only detected significant line emission in a few sources, namely X0614+091, Sco X-1, Cyg X-2, and Cyg X-3 (Christian 1993). Several bursters, e.g. $\mathrm{X} 1636-536, \mathrm{X} 1735-44$, and Ser X-1 had $3 \sigma$ detections based on line energies previously reported by the higher resolution OGS (Vrtilek et al. 1991).

\section{DISCUSSION}

A unique physical model does not emerge from the data, however at least two physically distinct regions are suggested. An optically thick boundary layer, which is well described as a 1-2 keV blackbody, and an optically thin, possibly extended region, which is well described by TB or a form of Comptonization. However the data can not distinguish between the standard two component models $(\mathrm{BB}+\mathrm{TB}$ or $\mathrm{BB}+\mathrm{USC})$ and models based on a spherically symmetric distribution of gas with varying optical depth (Lamb 1989; Ponman, Foster, and Ross 1990).

\section{REFERENCES}

Christian, D. J. 1993, Univ. of Maryland, Ph.D. Thesis

Lamb, F. K. 1989, Proc. 23rd ESLAB, Symposium 1, 215

Morris, R., and McCammon, D. 1983, ApJ, 270, 119

Parsignault, D. R., and Grindlay, J. E. 1978, ApJ, 225, 970

Ponman, T. J. 1982, MNRAS, 201, 769

Ponman, T. J., Foster, A. J., and Ross, R. R. 1990, MNRAS, 246, 287

Schulz, N. S., Hasinger, G., and Trumper, J. 1989, A\&A, 225, 48

Sunyaev, R. A. and Titarchuk, L. G. 1980, A\&A, 86, 121.

Swank, J. H., and Serlemitsos, P. J. 1985, in Galactic and Extragalactic Compact

X-ray Sources, ed. Y. Tanaka and W.H.G. Lewin (Tokyo:ISAS), 175

Vrtilek, S. D., McClintock, J. E., Seward. F. D., Kahn, S. M., and Wargelin, B. J. 1991, ApJS, 76, 1127

White, N. E., Stella, L., and Parmar, A. 1988, ApJ, 324, 363 